\title{
PRÉFACE DE L'ÉDITION FRANÇAISE
}

C'est un réel plaisir de présenter la traduction française de l'ouvrage du Professeur Stefan Kubik. Le texte et l'image sont associés avec bonheur dans cet atlas d'anatomie topographique du corps humain et la description s'accompagne ici d'heureuses considérations cliniques. L'originalité de ce livre réside surtout dans l'illustration: l'illustration est toujours importante, capitale, dans un livre d'anatomie. L'image est le vrai document, le texte, le commentaire. Un dessin est, cependant, une interprétation, le schéma qui en est la simplification s'éloigne davantage encore du modèle, sa fonction démonstrative est trop personnelle pour être objective. L'image vraie, celle du modèle, est avec évidence la photographie, mais en noir et blanc elle est peu lisible et nécessite des retouches; seule la couleur donne la représentation fidèle de la préparation anatomique, elle suggère le relief, recréant la profondeur, rendant sans truquage la vérité des rapports.

Le lecteur d'un ouvrage d'anatomie éprouve une profonde satisfaction à retrouver dans un livre ce qu'il a vu ou sait qu'il peut voir lui-même - avec la photographie en couleurs, le vrai et le beau peuvent coïncider. C'est le but que s'est fixé le Professeur Stefan Kubik: les photographies de son bel atlas restituent le volume, la couleur, les rapports des organes, ce que découvre la dissection dans ses approches successives et, ici, les préparations anatomiques sont excellentes. Un dessin accompagne les photographies; il est nécessaire, car la réalité est trop riche, il supprime les difficultés de l'identification par lecteur novice des éléments anatomiques sur l'image photographique.

Cette anatomie topographique est une anatomie médico-chirurgicale, elle rappelle chaque fois que cela est opportun l'intérêt de tel ou tel dispositif anatomique, d'un rapport ou d'une variation. Ces considérations d'ordre pratique sont souhaitées par l'étudiant désireux qu'on lui montre la place de l'anatomie dans sa formation médicale. Avec la description qu'accompagne un exposé des applications cliniques, l'anatomie entre dans la vie. Elle n'est pas, elle n'est plus, une science du cadavre.

Le projet du Professeur Stefan Kubik était ambitieux, sa réalisation ne mérite que des éloges.

\section{Dr André DELMAS}

Professeur d'Anatomie à la Faculté de Médecine de Paris 
